\title{
Open tibial fractures: risk factors for infection in conversion of external fixator to intramedullary nail at a tertiary academic hospital
}

\author{
Maku MM11, Ngcelwane MV², Olorunju SAS ${ }^{3}$ \\ 1 BSc, MBChB(UFS); Consultant orthopaedic surgeon, Department of Orthopaedic Surgery, Steve Biko Academic Hospital, University of Pretoria \\ ${ }_{2}^{2}$ MBChB, FCS(SA)Orth, MSc(Orth)London; Consultant orthopaedic surgeon, Department Orthopaedic Surgery, Steve Biko Academic Hospital, \\ University of Pretoria \\ ${ }^{3}$ BSc, MSc, PhD; Biostatistician, South African Medical Research Council, Pretoria
}

Corresponding author: Prof MV Ngcelwane, Department of Orthopaedic Surgery, Steve Biko Academic Hospital, University of Pretoria; email: mthunzi.ngcelwane@up.ac.za, tel: (012) 354 2851; fax: (012) 3542821

\begin{abstract}
Aim: To determine the rate of infection in open tibial fractures treated by conversion of an external fixation into an intramedullary nail, and to identify the factors contributing to the infections.

Methods: The study included a total of 52 patients. Multiple variables were assessed as risk factors that could lead to infection in open tibial fractures treated primarily with an external fixator and later converted into an intramedullary nail. The factors looked at were: age, average time taken from injury to debridement, average time taken from admission to debridement, antibiotics administration, facility that admitted the patient before intramedullary nail, average time for conversion of external fixator into intramedullary nail insertion, type of soft tissue management, initial Gustilo and Anderson classification and retrospective re-classification of fractures, existence of superficial sepsis or pin-tract infection, radiologic evidence of infection, the Injury Severity Score and the type of external fixator used. A p value $<0.05$ was used as the threshold for level of significance.
\end{abstract}

Results: The average follow-up was 37 weeks (median 24 weeks). We had a 40\% infection rate Cl [27\%, 55\%]. Factors that were found to be the most statistically significant $(p \leq 0.05)$ were amount of soft tissue injury and fracture comminution; this is after the fractures were retrospectively re-classified. All other factors looked at were not statistically significant as risk factors for infection ( $p>0.05)$.

Conclusion: The study suggests that correct classification of open tibial fractures, with recognition of soft tissue injury and bone comminution, is important in reducing infection rates and in ensuring proper initial management of these fractures. Treatment should be based on the classification done in theatre during the initial debridement, rather than on presentation in the trauma unit.

Level of evidence: Level 4

Key words: open tibial fractures, external fixation, intramedullary nailing, infection rate

Citation: Maku MM, Ngcelwane MV, Olorunju SAS. Open tibial fractures: risk factors for infection in conversion of external fixator to intramedullary nail at a tertiary academic hospital. SA Orthop J 2018;17(2):20-24. http://dx.doi.org/10.17159/2309-8309/2018/v17n2a3

Editor: Prof Anton Schepers, University of the Witwatersrand

Received: September 2017

Accepted: January 2017

Published: May 2018

Copyright: @ 2018 Maku MM, et al. This is an open-access article distributed under the terms of the Creative Commons Attribution Licence, which permits unrestricted use, distribution and reproduction in any medium, provided the original author and source are credited.

Funding: No external funding was received for conducting this study.

Conflict of interest: There is no conflict of interest. None of the authors have any relation to the companies whose implants were used in this study. 


\section{Background}

Open tibia fractures are common long bone fractures, often resulting in extensive bone and soft tissue damage. ${ }^{1}$ The subcutaneous location of the tibia as well as its poor blood supply makes it susceptible to non-unions and infections. ${ }^{2}$ Injuries to the neurovascular structures are also a known complication. ${ }^{1}$

Currently, the management of open tibial fractures comprises thorough wound debridement, immediate bone stabilisation with an external fixator, and coverage of bone with soft tissue. This aids in enabling early mobilisation and restoration of limb function. Because of the complications of external fixators, such as pin-tract sepsis and non-union, there is a trend to convert the external fixator into an intramedullary fixation device. ${ }^{2}$ This treatment is also not without its complications, infection being a major one.

Yokoyama et al. mention factors found to influence infection rates post-operatively. ${ }^{2}$ They include the patient's age, sex, severity of the fracture as classified by Gustilo and Anderson (GA), ${ }^{3}$ the fracture site, Injury Severity Score (ISS), time to debridement, reamed versus unreamed nailing, the duration of external fixation, interval between external fixation and intramedullary nail, time taken to achieve closure of skin, and the existence of superficial or pin-tract infection. ${ }^{2}$

In their original classification, Gustilo and Anderson classified open tibial fractures into three types: ${ }^{3}$

- Type I - an open fracture with a wound less than $1 \mathrm{~cm}$ long, clean wound

- Type II - an open fracture with a wound more than $1 \mathrm{~cm}$ long without extensive soft-tissue damage, flaps or avulsions

- Type III - an open fracture with extensive soft-tissue damage, or a traumatic amputation

Type III fractures were subsequently further subdivided into three subtypes, because of the problems that were observed in their management, notably high infection rates and amputations: ${ }^{4}$

- Type IIIA - adequate soft-tissue coverage of a fractured bone despite extensive soft-tissue laceration or flaps, or high-energy trauma irrespective of the size of the wound

- Type IIIB - extensive soft-tissue injury with periosteal stripping and bony exposure. This is usually associated with massive contamination.

- Type IIIC - open fracture-associated arterial injury requiring repair

Type III GA fractures pose a special challenge to the orthopaedic surgeon because of their soft tissue component, degree of contamination and fracture configuration.

Types IIIB and IIIC present with periosteal stripping, muscle contusions, contamination and neurovascular structure damage. It is therefore not recommended for these types of injuries to be nailed primarily. Irrigation, debridement, external fixator, antibiotics and consultation with the plastic surgeon for the method of closing the defect are recommended steps in the management of the types III $\mathrm{B}$ and $\mathrm{C}$ fractures. ${ }^{1,2}$ The literature on this topic shows that infection rates are still a concern regardless of the form of intervention. .,5, $^{2,6}$

At the orthopaedic outpatient clinic in Steve Biko Academic Hospital, there has been a number of patients presenting with nail sepsis after secondary intramedullary nailing of open tibial fractures. The contributing factors for sepsis in these patients are however not clear, and this prompted the undertaking of this study.

\section{Aim}

To determine the rate of infection in open tibial fractures treated by conversion of an external fixator into an intramedullary nail, and to identify the factors contributing to the infections.

\section{Patients and methods}

We reviewed the clinical records of all patients treated at Steve Biko Academic Hospital for open fractures of the tibia during the period January 2009 to December 2013. Convenience sampling was used to include patients into this retrospective, observational study. ${ }^{7}$ We included all skeletally mature patients with open diaphyseal fractures of the tibia, treated with an external fixator and later converted to a nail.

We identified 66 patient records. After excluding all patients with metaphyseal fractures, patients with diabetes and peripheral vascular diseases, pregnant patients, and patients with inadequate clinical records (total 14 patients), we were left with 52 patients, and these formed the basis of this study.

There were 46 male patients and six females. Their age range was $18-58$ years, with a mean age of 34 years. The mean follow-up duration was 37 weeks, with a median of 24 weeks.

The standard protocol in our hospital is that in patients who present with open tibia fractures, the wound is covered with a sterile dressing and the limb is immobilised with a temporary back slab in casualty. Antibiotics are commenced in casualty. The attending surgeon grades the fracture using the GA grading. ${ }^{3}$ For GA I and II open fractures, cefazolin $2 \mathrm{~g}$ stat is given intravenously, followed by $1 \mathrm{~g} 8$ hourly intravenously for $24-72$ hrs. For GA III open fractures, intravenous amoxicillin clavulanate $2.4 \mathrm{~g}$ loading dose, followed by $1.2 \mathrm{~g}$ intravenously 8 hourly, till soft tissue closure (7-10 days). Patients who are allergic to penicillin are given clindamycin $600 \mathrm{mg}$ intravenously. In this study, no patients were identified to have a penicillin allergy. Patients are given three doses of antibiotics for simple fractures post-operatively, extending to seven days for complex fractures.

Wound management entails primary debridement of the wound, removing all contaminating foreign material, all necrotic tissue and all loose bone fragments. The wound is then temporarily covered with a hydrogel wound dressing mixed with gentamycin, the dressing being the vector for the gentamicin and to keep the wound moist. If the treating surgeon assesses the injury to be a GA type I, primary wound closure is done.

The fracture is stabilised with an external fixator device (predominantly a Hoffman external fixator, Smith and Nephew).

The patient is taken back to theatre in 48-72 hours for re-assessment of the soft tissues and removal of the hydrogel dressing. Fractures with minimal comminution associated with soft tissues that can be treated with secondary closure, undergo secondary internal fixation with an intramedullary nail (Trigen nail, Smith and Nephew, Memphis, Tennessee). The 52 patients in this study belong to this group.

In GA types IIIA-IIIC, the temporary external fixator is converted to a definitive external fixator, that is, they do not undergo intramedullary nail insertion. These patients may also require other means of soft tissue cover, and as such require consultation with plastic surgeons.

The following data was collected from the files of the study patients: age, sex, GA classification recorded initially by the treating doctor, fracture site, ISS, time interval from injury to debridement, time interval from admission to debridement, type of external fixator used, duration in external fixator, type of soft tissue management, interval between external fixator and intramedullary nail, clinical and radiological evidence of infection. We used the criteria described by Fukushima et al. ${ }^{6}$ to define wound infection. In superficial wound infection, the infection lies entirely above the fascia with associated erythema and tenderness, which requires antibiotics and opening of the wound. Deep infection is defined as an infection involving bone, as well as the tissue below the fascia. Pin-tract sepsis is defined as persistent drainage from a pin site that requires intervention or positive bacterial cultures from the site. ${ }^{6}$ The following radiologic features were used as indicators of infection: periosteal thickening, 
lytic lesions, endosteal scalloping and new bone apposition, implant loosening as indicated by lucency around the intramedullary tibial nail associated with cortical thinning, cortical irregularity and subperiosteal new bone formation. ${ }^{8}$

During data collection, when looking at the description of the wound, soft tissue damage and periosteal stripping, and reviewing the extent of comminution of the fractures on X-ray $(X R)$, we found some fractures that were initially classified as GA I which were actually GA II , some that were classified as GA II fractures which were actually GA IIIA and some GA IIIA whose severity of injury was also underestimated. We did not have any GA IIIC in the study.

The data was recorded onto a data-capturing sheet, which was used to perform statistical analysis. Variables collected were tested against each other to determine their level of significance in being a risk factor for infection.

\section{Statistical analysis}

Stata 14.1 statistical software was used to analyse the data. ${ }^{9}$ The analysis presented descriptive statistics by demographic characteristics, including proportions, standard errors and associated 95\% confidence intervals. An independent t-test for comparing proportions was used to compare various categories between infected and non-infected groups. Chi-square analysis was used to determine the association between the rates of infection and the associated factors. Testing was done at the 0.05 level of significance $(p<0.05)$

\section{Results}

The patients were divided into two groups. Group A comprised those that were assessed as being not infected at final follow-up. Group B comprised those that were complicated by infection. The results are summarised in Table I.

Out of the 52 fractures, 21 (40\%) were complicated by infection. The average time from injury to debridement was $27 \mathrm{hrs}$ in Group A and 22 hrs in Group B. The time spent in hospital before theatre was 18 hrs in Group A and 17 hrs in Group B.

After initial debridement, most of the patients were nursed in the orthopaedic ward (28 in Group A and 20 in Group B). A few of the patients, because of associated injuries, were managed in ICU (two in Group A and one in Group B) and one from Group A was managed in a high care ward.

In 14 patients primary wound closure was performed; six of these became septic. Secondary wound closure was performed in 28 patients; nine of these became septic. Six patients had split skin graft; four of them became septic. Four patients had gastrocnemius/soleus flap; two of them became septic. The average time to conversion of

Table I: Variables tested as contributors to infection in open tibial fractures

\begin{tabular}{|c|c|c|c|}
\hline Variable & Non-infected fractures (Group A) ( $n=31)$ & Infected fractures (Group B) (n=21) & P-values \\
\hline Average time from injury to debridement & 27 hours (3-84 hrs) & 22 hours $(7-120 \mathrm{hrs})$ & NS \\
\hline $\begin{array}{l}\text { Average time from admission to } \\
\text { debridement }\end{array}$ & 18 hours (0.6-48 hrs) & 17 hours (2-72 hrs) & NS \\
\hline Type of external fixator used & $\begin{array}{c}\text { Hoffman ex-fix }=27 \\
\text { Dispofix }=1 \\
\text { Orthofix }=2 \\
\text { Jet- } x=1\end{array}$ & $\begin{array}{c}\text { Hoffman ex-fix }=17 \\
\text { Dispofix }=2 \\
\text { Orthofix }=2 \\
\text { Jet- } x=0\end{array}$ & NS \\
\hline $\begin{array}{l}\text { Admitting facility between external fixator } \\
\text { and intramedullary nail }\end{array}$ & $\begin{array}{c}\text { Ward }=28 \\
I C U=2 \\
\text { High care }=1\end{array}$ & $\begin{array}{c}\text { Ward }=20 \\
I C U=2 \\
\text { High care }=0\end{array}$ & NS \\
\hline $\begin{array}{l}\text { Wound coverage } \\
\text { Primary closure } \\
\text { Secondary cover } \\
\text { Split skin graft } \\
\text { Flap (gastrocnemius/soleus) }\end{array}$ & $\begin{array}{c}8 \\
19 \\
2 \\
2\end{array}$ & $\begin{array}{l}6 \\
9 \\
4 \\
2\end{array}$ & NS \\
\hline $\begin{array}{l}\text { Average time to conversion of external } \\
\text { fixator to an intramedullary nail }\end{array}$ & $\begin{array}{l}5.7 \text { days }(2-19) \\
\text { Median } 3 \text { days }\end{array}$ & $\begin{array}{l}3.4 \text { days }(1-9) \\
\text { Median } 4.3 \text { days }\end{array}$ & NS \\
\hline Injury Severity Score & $\mathrm{n}=17(5-36)$ & $\mathrm{n}=19(9-27)$ & NS \\
\hline
\end{tabular}

${ }^{*} \mathrm{NS}=$ not statistically significant

Table Ila: Gustilo and Anderson classification of fractures as contributor to infection

\begin{tabular}{|c|c|c|c|}
\hline GA classification & Non-infected (Group A) $(n=31)$ & Infected (Group B) $(n=21)$ & $p$-values \\
\hline I $(n=8)$ & $4 / 8$ & $4 / 8$ & $p=0.01$ \\
II $(n=35)$ & $22 / 35$ & $13 / 35$ & $p>0.05$ \\
IIIa $(n=8)$ & $3 / 8$ & $5 / 8$ & $p>0.05$ \\
IIIb $(n=1)$ & $1 / 1$ & $0 / 1$ & $p>0.05$ \\
III $(n=0)$ & $0 / 0$ & $0 / 0$ & - \\
\hline
\end{tabular}

Table Ilb: Gustilo and Anderson classification as contributor to infection after reclassifying the fractures

\begin{tabular}{|c|c|c|c|}
\hline GA classification & Non-infected (Group A) $(n=31)$ & Infected (Group B) $(n=21)$ & $p$-values \\
\hline I $(n=4)$ & $4 / 4$ & $0 / 4$ & $p=0.005$ \\
II $(n=26)$ & $22 / 26$ & $4 / 26$ & $p=0.000$ \\
IIIa $(n=20)$ & $3 / 20$ & $16 / 20$ & $p=0.000$ \\
IIIb $(n=2)$ & $2 / 2$ & $1 / 2$ & $p=1.0$ \\
III $(n=0)$ & $0 / 0$ & $0 / 0$ & - \\
\hline
\end{tabular}


external fixator to intramedullary nail was 5.7 days for Group A, and 3.4 days for Group B.

As seen in Table I, the following factors were not significant in causing sepsis, as indicated by $p$-values exceeding 0.05: average time from injury to debridement, average time from admission to debridement, average time to conversion of external fixator to an intramedullary nail, type of external fixator used, type of ward to which patient is admitted between the external fixator and intramedullary nail period, and wound coverage.

Table // looks at the results after re-classifying the fractures. The treating surgeons had initially classified 43 patients as being GA types I and II, and nine patients as being type III. On re-classifying the fractures, we found that 30 patients were types I and II, and 22 were type III.

Figure 1 is an example of a fracture that was initially graded as GA I, on the basis of a small skin wound of less than a centimetre. The theatre notes described that there was periosteal stripping. The XR showed a comminuted fracture. Combining all these we re-classified this injury as GA IIIA.

On the initial classification of the fractures, there was no significant difference in infection rates between the GA types of the fractures, as seen in Table lla.

However, upon re-classification of these fractures, there was a significant difference in infection rates between the various GA types of fractures, as seen in Table $/ / b$.

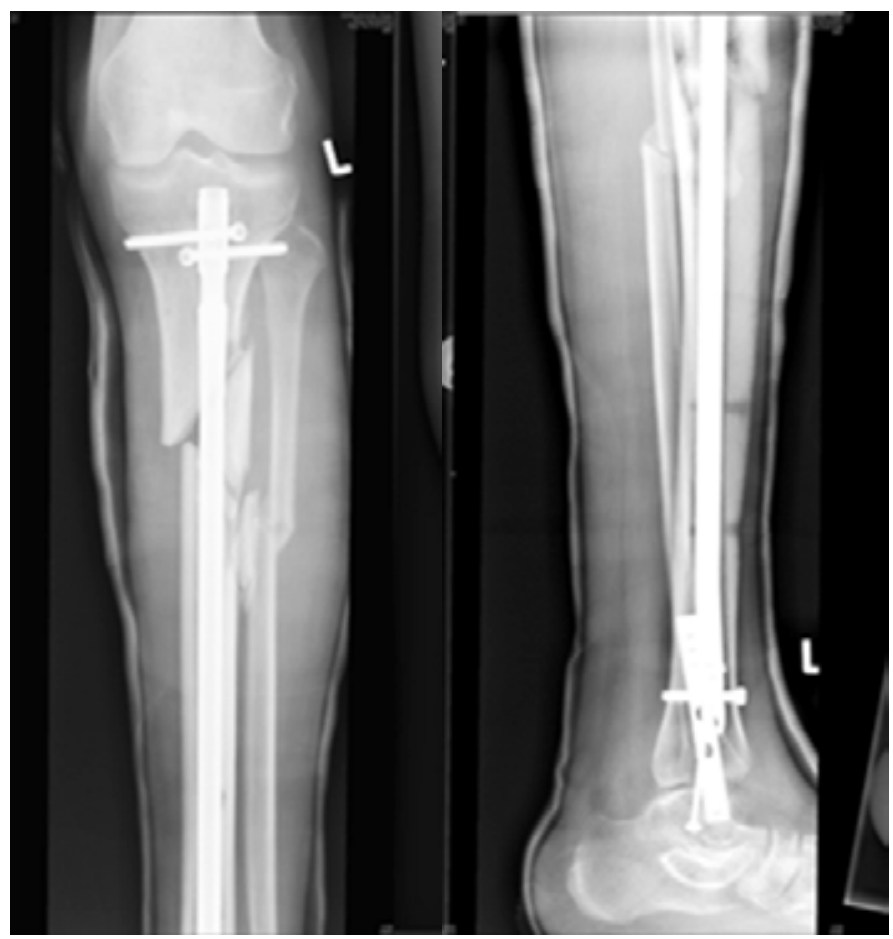

Figure 1. An example of a comminuted tibial fracture that was initially graded as GA I on the basis of a small puncture wound, and retrospectively re-graded to GA Illa. The fracture subsequently developed osteomyelitis.

\section{Discussion}

Open tibia fractures continue to have a high infection rate, reported to be $10-50 \%$ in the literature, 4,5 despite advances in wound care, soft tissue cover and types of internal and external fixator devices. This study shows an infection rate of $40 \%$, which is comparable to the literature.

According to Bashir et al., ${ }^{1}$ the treatment of choice for GA types I to III open tibia fracture is an external fixator. With the advent of the interlocking nail, intramedullary nailing has become a very popular treatment choice in tibia fracture. The success of the intramedullary nail is related to the high union rates, reduced requirements for bone grafting, decreased hospital stays, low incidences of mal-union, and rapid return to normal function. Our hospital follows a similar protocol when managing open tibial fractures.

There is no consensus that debriding open fractures within 6 hours helps to reduce infection rates. ${ }^{10,11}$ There is however literature that shows that late debridement, beyond 6 hours, does increase infection rates. ${ }^{10,12}$ In this study, the average time from injury to debridement was 27 hours and 22 hours in Group A and Group B respectively. However, this was not statistically significant. The average time from admission to debridement was 18 hours and 17 hours for Group $A$ and $B$ respectively. This was not statistically significant, despite the long wait the patients had in hospital before debridement. We attribute this result to our pre-debridement antibiotic policy. This is well documented by other authors who show that early administration of antibiotics is vital in preventing infection. 5,10,13

The time interval between external fixation and intramedullary nail insertion varies in the literature. ${ }^{14}$ It is also dependent on the type of fracture that the surgeon is faced with. Various authors recommend waiting an average of 9-11 days before introducing the nail to the medullary canal, and then using specific clinical indicators such as waiting for granulation tissue circumscribing the pin tract sites before intramedullary nailing. ${ }^{12,14}$ For the type IIIA fracture, a waiting period of 15 days has been shown to be beneficial after debridement and systemic antibiotic administration. ${ }^{12}$

In this study there was a mean waiting period of 5.7 days for the non-infected group, and 3.4 days for the infected group ( $p>0.05$ ). The infected group was taken to theatre for a definitive intramedullary nail insertion a day and a half earlier than the non-infected group. There was no statistical significance in this result.

The type of temporary external fixator used did not prove to be a significant risk factor for the increased incidence of infection.

Trampuz and Zimmerli state that microbial contamination occurs in delayed wound closure. ${ }^{15}$ Early wound coverage and bone grafting forms part of aggressive soft tissue management, which plays a major role in open tibia fracture treatment. ${ }^{16}$ Yokoyama found that skin closure within one week reduces the incidence of deep infections when treating open tibial fractures with an intramedullary nail.?

The definition of pin-tract sepsis entails any persistent drainage from a pin site that will require an intervention, or a positive bacterial culture from the pin site. ${ }^{2}$ The use of external fixators is associated with a high incidence of pin-tract sepsis. The incidence of infection is then increased when sequential nailing is undertaken, even if adequate antibiotic coverage is provided. ${ }^{6}$ Wheelwright and CourtBrown found that if sequential nailing is undertaken after granulation tissue has formed along the pin-tract, the incidence of infection may be reduced. ${ }^{12}$ In this study 44 patients had superficial sepsis and pin-tract infection. They did not, however, progress to osteomyelitis. Eight patients progressed to osteomyelitis after a superficial sepsis and pin-tract infection.

The ISS has been suggested as a predictor for deep infection in open tibia fractures. ${ }^{17}$ In the study done by Yokoyama et al. ${ }^{2}$, it was found that there was no significant difference among patients with a high or low ISS in the incidence of deep infection. This corresponds with the results of our study, where we also found that the ISS was not a statistically significant factor in contributing to deep infection in open tibia fractures.

This study provides evidence that soft tissue injury and comminution of open tibia fractures are the leading factors in determining the outcome of infection in open tibial fractures treated initially with an external fixator and converted to an intramedullary nail. This amount of soft tissue injury and bone comminution was statistically significant $(p=0.05)$.

When the treating surgeons classified the fracture, they classified them into GA I, II and III without taking account of the comminution and soft tissue injury. This showed no statistical relevance in the 
cause of infection, as shown in Table lla. In this study, we re-classified the fractures ${ }^{4}$ taking into account the amount of soft tissue injury and periosteal stripping as detailed in the operation notes, and the comminution of the fracture as seen on the radiographs. This then showed a statistically significant relationship between the GA classification and the rate of deep infections in these open tibial fractures as seen in Table Ilb. This has also been shown by different authors in the literature. ${ }^{17-20}$

The extent of soft tissue injury, periosteal stripping and bone comminution is an indication of the amount of energy transferred to the limb during the injury. We consider it a significant finding that this correlates much more with infection than other factors we examined in this study. In particular, it is important to note that the amount of energy transferred to the patient in general as shown by the ISS does not correlate very well to infection in this study. This suggests that the interruption of blood supply to the bone as evidenced by the amount of soft tissue injury and periosteal stripping may be the main factors responsible for infection.

\section{Limitations of the study}

Being a retrospective study, we lost some patients because of inadequate records.

\section{Conclusion}

The study shows that the main factor contributing to infection in open fractures of the tibia is the extent of energy transferred to the limb, as expressed by periosteal stripping and comminution of the fracture as seen on the XR.

Treatment should be based on the Gustilo and Anderson classification done during the initial surgery of wound debridement, and not on presentation in the trauma unit.

\section{Ethical consideration}

No benefits of any form have been received by any of the authors from a commercial party related to the subject of this study. Ethical approval was obtained from the ethics committee of Health Sciences of the University of Pretoria (Ethics Ref No: 112/2016).

\section{References}

1. Bashir A, Dar TA, Badoo AR, Ganie MA. Secondary intramedullary nailing after primary external fixation in the treatment of tibial fractures. The Internet Journal of Orthopedic Surgery. 2009; 12(1):1-3.

2. Yokoyama $\mathrm{K}$, et al. Risk factors for deep infection in secondary intramedullary nailing after external fixation for open tibial fractures. Injury, Int J Care Injured. 2006;37:554-60.

3. Gustilo RB, Anderson JT. Prevention of infection in the treatment of one thousand and twenty-five open fractures of long bones: retrospective and prospective analysis. $J$ Bone Joint Surg $[\mathrm{Am}]$. 1976;58(A):453-58.

4. Gustilo RB, Mendoza RM, Williams DN. Problems in the management of type III (severe) open fractures: A new classification of type III open fractures. J Trauma.1984;24(8):742-46.

5. Shorin HR, et al. Antibiotic prophylaxis in bacterial infection of type IIIA open fracture of tibial shaft with or without fibula fracture. Ravazi Int J Med. 2016 June;4(2):1-7.
6. Fukushima, et al. Immediate versus delayed intramedullary nailing for open fractures of the tibial shaft; A multivariate analysis of factors affection deep infection and fracture healing. Indian Journal of Orthopaedics. 2008;42(4):410-17.

7. Golafshani N. Understanding reliability and validity in qualitative research. The Qualitative Report. December 2003;8(4):597-604.

8. Bhat V, Gupta $H$. The radiological diagnosis of infection. Orthopaedics and Trauma. 2014;28(6):409-16.

9. StataCorp. Stata: Release 14. Statistical software. Texas: College Station, StataCorp LP. 2014.

10. Penn-Barwell JG, Murray CK, Wenke JC. Early antibiotics and debridement independently reduce infection in an open fracture model. J Bone Joint Surg [Br] 2012;94(B):107-12.

11. Crowley DJ, Kanakaris NK, Giannoudis PV. Debridement and wound closure of open fractures: The impact of the time factor on infection rates. Injury, Int J Care 2007;38:879-89.

12. Wheelwright EF, Court-Brown CM. Primary external fixation and secondary intramedullary nailing in the treatment of tibial fractures. Injury. 1992;23(6):373-76.

13. Sharr PJ, Buckley RE. Current concepts review: open tibial fractures. Arch Orthop Trauma Surg. 2014;81:95-107.

14. Vikas K. Incidence of infection after early intramedullary nailing of open tibial shaft fractures stabilized with pinless external fixators. Indian Journal of Orthopaedics. 2008;42(4):401-407.

15. Trampuz A, Zimmerli W. Diagnosis and treatment of infections associated with fracture-fixation devices. Injury, Int J Care Injured. 2006;37:S59-S66.

16. Sienbenrock KA, Gerich T, Jakob RP. Sequential intramedullary nailing of open tibial shaft fractures after external fixation. Arch Orthop Trauma Surgery 1997;116:32-36.

17. Metsemakers WJ, Handojo K, Reynders P, Sermon A, Vanderschot $\mathrm{P}$, Nijs S. Individual risk factors for deep infection and compromised fracture healing after intramedullary nailing of tibial shaft fractures: A single centre experience of 480 patients. Injury, Int J Care Injured. 2015;46:740-45.

18. Roussignol X, Sigonney G, Potage D, Ettienne M, Duparc F, Dujardin F. Secondary nailing after external fixation for tibial shaft fracture: Risk factors for union and infection. A 55 case series. Orthopaedic and Traumatology; Surgery and Research 2015;101:89-92.

19. Papakostidis C, et al. Prevalence of complications of open tibial shaft fractures stratified as per the Gustilo-Anderson classification. Injury, Int J Care Injured. 2011;42:1408-15.

20. Khatod, et al. Outcomes in open tibia fractures; Relation between delay in treatment and infection. Journal of Trauma and Injury, Infection, and Critical Care. 2003;55:949-54. 